Math. Model. Nat. Phenom.

Vol. 5, No. 4, 2010, pp. 32-53

DOI: $10.1051 / \mathrm{mmnp} / 20105402$

\title{
Discrete Spectrum of the Periodic Schrödinger Operator with a Variable Metric Perturbed by a Nonnegative Potential
}

\author{
M. Sh. Birman, V. A. Sloushch* ${ }^{\dagger}$ \\ Department of Physics, St. Petersburg State University, \\ Ul'yanovskaya 3, Petrodvorets, St. Petersburg, 198504, RUSSIA
}

\begin{abstract}
We study discrete spectrum in spectral gaps of an elliptic periodic second order differential operator in $L_{2}\left(\mathbb{R}^{d}\right)$ perturbed by a decaying potential. It is assumed that a perturbation is nonnegative and has a power-like behavior at infinity. We find asymptotics in the large coupling constant limit for the number of eigenvalues of the perturbed operator that have crossed a given point inside the gap or the edge of the gap. The corresponding asymptotics is power-like and depends on the observation point.
\end{abstract}

Key words: periodic Schrödinger operator, discrete spectrum, spectral gaps, asymptotics in the large coupling constant limit.

AMS subject classification: 47F05, 47G10.

\section{Introduction. Main result}

\subsection{Main goal of the paper. Notation}

Let $A$ be an elliptic periodic second order differential operator in $L_{2}\left(\mathbb{R}^{d}\right), d \geqslant 1$, and let $V$ be the operator of multiplication by a nonnegative function $V(x)$ decaying as $|x| \rightarrow+\infty$ (in appropriate sense). We study discrete spectrum of the perturbed operator $B(t):=A+t V$, $t>0$, in the spectral gaps of $A$. The sign of a perturbation is essential both for the kind

\footnotetext{
${ }^{*}$ E-mail: vsloushch@list.ru

${ }^{\dagger}$ Supported by RFBR (grant no. 08-01-00209-a).
} 
of results and the choice of technical tools. The case of nonpositive perturbations is studied much more completely than the case of nonnegative perturbations (see the survey [1] and references therein). The present paper is devoted to the case of nonnegative perturbations.

Let $(\alpha, \beta)$ be an internal gap in the spectrum of $A$, and let $\lambda \in[\alpha, \beta]$ be a fixed number called an observation point. The spectrum of the operator $B(t)$ in the gap $(\alpha, \beta)$ is discrete. As $t$ grows, the eigenvalues of the operator $B(t)$ in the gap $(\alpha, \beta)$ move from the left to the right; they "are born" at the left edge and they "disappear" at the right edge of the gap. By $N(\lambda, \tau, V)$ we denote the number of eigenvalues of the operator $B(t)$ that have crossed the point $\lambda$ as $t$ grows from 0 to $\tau$. In the survey [1], the asymptotics for $N(\lambda, \tau, V)$ as $\tau \rightarrow+\infty$ was described (without proof) in the case where the perturbation $V(x)$ has a power like asymptotics at infinity (see Theorem 1.2). The main goal of the paper is to prove this asymptotics for the case of "slowly" decaying perturbations (see Theorem 1.3).

The paper consists of three sections. Section 1 contains necessary notions and notation, formulation of the main result and discussion of the results known before. In Section 2, the main result (Theorem 1.3) is deduced from Proposition 2.3 about asymptotics of singular numbers of some integral operators. In Section 3, the required results on asymptotics of the singular numbers of integral operators are collected and Proposition 2.3 is proved.

Now we introduce some notation. The symbol 1 stands for the identity $(d \times d)$-matrix; $\langle\cdot, \cdot\rangle$ denotes the standard inner product in $\mathbb{C}^{d}$. By $\mathbb{Z}^{d}$ we denote the lattice of integer vectors in $\mathbb{R}^{d}$. Next, $H^{1}(\Omega)$ denotes the standard Sobolev class in a domain $\Omega \subset \mathbb{R}^{d}$, and $H_{\text {loc }}^{1}(\Omega)$ is the class of functions $u(x), x \in \Omega$, such that $u \varphi \in H^{1}(\Omega)$ for any $\varphi \in C_{0}^{\infty}(\Omega)$. By $\Delta$ we denote the Laplacian in $\mathbb{R}^{d}$. If $A$ is a closed densely defined operator in the Hilbert space $\mathfrak{H}$, then $\sigma(A)$ stands for its spectrum, and $A^{*}$ stands for the adjoint operator. If $A$ is selfadjoint, then $E_{A}(\cdot)$ denotes the spectral measure of $A$. For a Borel set $\delta \subset \mathbb{R}$, we put $\pi_{A}(\delta):=\operatorname{rank} E_{A}(\delta)$. Next, we introduce the counting functions for the positive and negative spectrum of $A$ by $n_{ \pm}(s, A):=\pi_{ \pm A}(s,+\infty), s>0$. By $\mathfrak{B}(\mathfrak{H})$ we denote the class of bounded linear operators in $\mathfrak{H}$; the symbol $\mathfrak{S}_{\infty}(\mathfrak{H})$ stands for the class of compact linear operators in $\mathfrak{H}$.

\subsection{The unperturbed operator}

Let $g$ be a $(d \times d)$-matrix-valued function with real entries, and let $p$ be a real-valued potential such that

$$
\begin{gathered}
p, g \in L_{\infty}\left(\mathbb{R}^{d}\right), \quad g(x) \geqslant c_{0} \mathbf{1}, \quad c_{0}>0, \\
g(x+n)=g(x), \quad p(x+n)=p(x), \quad n \in \mathbb{Z}^{d} .
\end{gathered}
$$

Consider the operator

$$
A=-\operatorname{div} g(x) \operatorname{grad}+p(x)=\nabla^{*} g(x) \nabla+p(x),
$$

acting in the Hilbert space $\mathfrak{H}=L_{2}\left(\mathbb{R}^{d}\right), d \geqslant 1$. Precisely, $A$ is a selfadjoint operator in $\mathfrak{H}$ generated by the closed semibounded quadratic form

$$
a[u, u]:=\int_{\mathbb{R}^{d}}\left(\langle g(x) \nabla u, \nabla u\rangle+p(x)|u|^{2}\right) d x, \quad u \in H^{1}\left(\mathbb{R}^{d}\right) .
$$


In order to describe the spectrum of $A$, we introduce standard objects related to the spectral decomposition of a periodic elliptic operator (see, e. g., [2], [3]). Let $\Omega:=[0,1)^{d}$ be the cell of the lattice $\mathbb{Z}^{d}$, and let $\widetilde{\Omega}:=[-\pi, \pi)^{d}$ be the cell of the dual lattice $(2 \pi \mathbb{Z})^{d}$. In $L_{2}(\Omega)$, we consider a family of selfadjoint operators $A(k), k \in \widetilde{\Omega}$. The operator $A(k)$ is defined in terms of the quadratic form

$$
a(k)[u, u]:=\int_{\Omega}\left(\langle g(x) \nabla u, \nabla u\rangle+p(x)|u|^{2}\right) d x, \quad u \in \operatorname{dom} a(k), \quad k \in \widetilde{\Omega} .
$$

The domain $\operatorname{dom} a(k), k \in \widetilde{\Omega}$, consists of all functions $u \in H^{1}(\Omega)$ such that the periodic extension of $e^{-i k x} u(x)$ belongs to $H_{\mathrm{loc}}^{1}\left(\mathbb{R}^{d}\right)$. The spectrum of $A(k)$ is discrete. Let

$$
E_{1}(k) \leqslant E_{2}(k) \leqslant \ldots \leqslant E_{n}(k) \leqslant \ldots
$$

denote the consecutive eigenvalues of $A(k)$. The mappings $E_{s}: \widetilde{\Omega} \rightarrow \mathbb{R}$ are called band functions. They extend periodically (with respect to the lattice $\left.(2 \pi \mathbb{Z})^{d}\right)$ to continuous functions in $\mathbb{R}^{d}$. Let $\left\{\psi_{s}(k, \cdot)\right\}_{s=1}^{\infty}, k \in \widetilde{\Omega}$, be the eigenfunctions of $A(k)$ which are orthonormal in $L_{2}(\Omega)$ and correspond to the eigenvalues (1.5). We extend functions $\psi_{s}(k, x)$ for all $x \in \mathbb{R}^{d}$ so that the functions $e^{-i k x} \psi_{s}(k, x)$ are $\mathbb{Z}^{d}$-periodic in $x \in \mathbb{R}^{d}$ for all $k \in \widetilde{\Omega}, s \in \mathbb{N}$. Note that (see, e. g., [4])

$$
\psi_{s}(\cdot, \cdot) \in L_{\infty}\left(\widetilde{\Omega} \times \mathbb{R}^{d}\right) .
$$

Now we introduce the operators $\Phi_{s}$ :

$$
\left(\Phi_{s} u\right)(k)=(2 \pi)^{-d / 2} \int_{\mathbb{R}^{d}} \overline{\psi_{s}(k, x)} u(x) d x, \quad u \in \mathcal{S}\left(\mathbb{R}^{d}\right), \quad s \in \mathbb{N} .
$$

The operator $A$ is partially diagonalized with the help of operators $\Phi_{s}$. Here $\mathcal{S}\left(\mathbb{R}^{d}\right)$ is the Schwartz class. The operators (1.6) are partially isometric mappings of $L_{2}\left(\mathbb{R}^{d}\right)$ onto $L_{2}(\widetilde{\Omega})$. The operators $\Phi_{s}^{*} \Phi_{s}=: P_{s}, s \in \mathbb{N}$, are mutually orthogonal projections in $L_{2}\left(\mathbb{R}^{d}\right)$, and $\sum_{s \in \mathbb{N}} P_{s}=I$. The operator $A$ admits the Floquet decomposition:

$$
A=\sum_{s \in \mathbb{N}} \Phi_{s}^{*}\left[E_{s}(k)\right] \Phi_{s}
$$

Here and in what follows the symbol $[m(k)]$ stands for the operator of multiplication by the function $m(k)$ in $L_{2}(\widetilde{\Omega})$. The spectrum of $A$ is the union of the spectral bands that are the images of the continuous functions $E_{s}$ :

$$
\sigma(A)=\bigcup_{s=1}^{\infty} \operatorname{Im} E_{s} .
$$

The spectrum $\sigma(A)$ may contain gaps. Assume that $(\alpha, \beta)$ is a gap in $\sigma(A)$. Then for some $l \geqslant 2$ we have

$$
\alpha=\max _{k \in \widetilde{\Omega}} E_{l-1}(k)<\beta=\min _{k \in \widetilde{\Omega}} E_{l}(k) .
$$




\subsection{Perturbation}

The operator (1.3) is perturbed by a real-valued potential $V(x)$. Assume that $V(x)$ satisfies the following conditions:

$$
\begin{gathered}
V(x) \geqslant 0, \quad V \in L_{\infty}\left(\mathbb{R}^{d}\right), \\
V(x)=\omega(x /|x|)|x|^{-\varrho}+o\left(|x|^{-\varrho}\right), \quad|x| \rightarrow+\infty, \quad \varrho>0 .
\end{gathered}
$$

Under conditions (1.9), (1.10), the function $\omega$ is nonnegative and bounded on $\mathbb{S}^{d-1}$. Since the domain of the quadratic form (1.4) coincides with $H^{1}\left(\mathbb{R}^{d}\right)$, we have

$$
(-\Delta+I)^{1 / 2}(A-\zeta I)^{-1 / 2} \in \mathfrak{B}(\mathfrak{H}), \quad \zeta<\inf \sigma(A) .
$$

Besides, under conditions $(1.9),(1.10)$ the operator $\sqrt{V}(-\Delta+I)^{-1 / 2}$ is compact (e. g., this follows from the results of [5]). Hence, we have

$$
\sqrt{V}(A-\zeta I)^{-1 / 2} \in \mathfrak{S}_{\infty}(\mathfrak{H}), \quad \zeta<\inf \sigma(A) .
$$

The perturbed operator is defined by $B(t):=A+t V, t>0$. By (1.9) and (1.11), the perturbation is nonnegative and relatively compact:

$$
\begin{gathered}
B(t) \geqslant A, \quad t>0 \\
(B(t)-i I)^{-1}-(A-i I)^{-1} \in \mathfrak{S}_{\infty}(\mathfrak{H}), \quad t>0 .
\end{gathered}
$$

Hence, the spectrum of the operator $B(t), t>0$, in the gap $(\alpha, \beta)$ is discrete and does not accumulate to the right edge of the gap. We consider the counting function

$$
N(\lambda, \tau, V):=\sum_{t \in(0, \tau)} \operatorname{dim} \operatorname{Ker}(B(t)-\lambda I), \quad \tau>0, \quad \lambda \in(\alpha, \beta) .
$$

By definition, the right-hand side of (1.12) is equal to infinity if there exist infinitely many points $t \in(0, \tau)$ such that $\operatorname{dim} \operatorname{Ker}(B(t)-\lambda I)>0$. The next statement (the BirmanSchwinger principle) follows from variational arguments (see, e. g., [6]).

Proposition 1.1. Suppose that conditions (1.1), (1.9), and (1.10) are satisfied. Assume also that $\sigma(A) \cap(\alpha, \beta)=\varnothing$. Then we have

$$
\begin{gathered}
Z(\lambda):=\sqrt{V}(\lambda I-A)^{-1} \sqrt{V} \in \mathfrak{S}_{\infty}(\mathfrak{H}), \quad \lambda \in(\alpha, \beta), \\
N(\lambda, \tau, V)=n_{+}\left(\tau^{-1}, Z(\lambda)\right), \quad \tau>0, \quad \lambda \in(\alpha, \beta) .
\end{gathered}
$$

From Proposition 1.1 it follows that $N(\lambda, \tau, V)$ is finite for all $\tau>0$ and $\lambda \in(\alpha, \beta)$, and is a nonincreasing function of $\lambda$ (for a fixed $\tau>0$ ). Now we define the counting function at the left edge of the gap:

$$
N(\alpha, \tau, V):=\lim _{\lambda \rightarrow \alpha+0} N(\lambda, \tau, V), \quad \tau>0 .
$$

The limit in the right-hand side can be finite or infinite. Naturally, $N(\alpha, \tau, V)$ can be interpreted as the number of eigenvalues of $B(t)$ that "were born" at the left edge of the gap as $t$ grows from 0 to $\tau$. 


\subsection{Main result}

Now we describe the asymptotics of the function $N(\lambda, \tau, V)$ as $\tau \rightarrow+\infty$. We put

$$
\Gamma_{\varrho}(\lambda):=(2 \pi)^{-d} d^{-1} \sum_{s=1}^{l-1} \int_{\widetilde{\Omega}}\left(\lambda-E_{s}(k)\right)^{-d / \varrho} d k \int_{\mathbb{S}^{d-1}} \omega^{d / \varrho}(\theta) d S(\theta), \quad \lambda \in[\alpha, \beta] .
$$

If $\lambda \in(\alpha, \beta)$, then $\Gamma_{\varrho}(\lambda)$ is a fortiori finite. Next, $\Gamma_{\varrho}(\alpha)$ is finite under the following conditions:

$$
\left(\alpha-E_{s}(\cdot)\right)^{-1} \in L_{d / \varrho}(\widetilde{\Omega}), \quad s=1, \ldots, l-1 .
$$

In the survey [1], the following theorem was formulated (without proof) in the case $d \geqslant 2$.

Theorem 1.2. Suppose that conditions (1.1), (1.2), (1.8), (1.9), and (1.10) are satisfied. Then for all $\lambda \in(\alpha, \beta)$ we have

$$
\lim _{\tau \rightarrow+\infty} \tau^{-d / \varrho} N(\lambda, \tau, V)=\Gamma_{\varrho}(\lambda)
$$

Under the additional condition (1.14) asymptotics (1.15) is valid also in the point $\lambda=\alpha$.

Our goal is to give the proof of Theorem 1.2 under the condition $\varrho \in(0, d), d \geqslant 1$, $\lambda \in[\alpha, \beta)$. In the case where $\lambda \in(\alpha, \beta)$ and $g(x)=\mathbf{1}$, Theorem 1.2 was proved in the pioneer paper [7]. In [7], the potential $p$ was not assumed to be periodic; it was only assumed that there exists the so called integrated density of states for the operator $A=-\Delta+p$. The case where $g(x)=\mathbf{1}, p(x)$ is periodic and $\lambda=\alpha$ was considered in [8].

In the present paper we establish the following result.

Theorem 1.3. Suppose that conditions (1.1), (1.2), (1.8), (1.9), and (1.10) are satisfied. Let $\varrho \in(0, d), d \geqslant 1$. Then for all $\lambda \in(\alpha, \beta)$ asymptotics (1.15) is valid. If, in addition, condition (1.14) is satisfied, then asymptotics (1.15) is true also for $\lambda=\alpha$.

In Section 2, the proof of Theorem 1.3 will be reduced to the study of asymptotics for singular numbers of some integral operators (which will be studied in Section 3). The order of asymptotics (1.15) differs from the "standard" order $\tau^{d / 2}$. The asymptotic coefficient depends on the observation point $\lambda$. However, it becomes clear that this asymptotics is of the "Weyl" nature, if the roles of coordinates and (quasi)momentums change.

We say that the left edge of the gap $(\alpha, \beta)$ is regular if $\max _{k} E_{l-2}(k)<\alpha, k \in \widetilde{\Omega}$, the equality $E_{l-1}(k)=\alpha$ is reached in a finite number of points $k_{j} \in \widetilde{\Omega}, j=1, \ldots, M$, and each $k_{j}$ is a point of nondegenerate maximum for $E_{l-1}$, i. e.,

$$
\alpha-E_{l-1}(k)=q_{j}\left(k-k_{j}\right)+O\left(\left|k-k_{j}\right|^{3}\right), \quad k \rightarrow k_{j}, \quad j=1, \ldots, M,
$$

where $q_{j}$ is a positive definite quadratic form.

If the left edge of the gap is regular, then

$$
\left(\alpha-E_{s}(\cdot)\right)^{-1} \in L_{q}(\widetilde{\Omega}), \quad s=1, \ldots, l-1, \quad \forall q<d / 2 .
$$


Thus, Theorem 1.3 implies the following statement.

Corollary 1.4. Let $d \geqslant 3$. Suppose that conditions (1.1), (1.2), (1.8), (1.9), and (1.10) are satisfied. Assume also that $\varrho \in(2, d)$, and the left edge of the gap is regular. Then asymptotics (1.15) is valid for $\lambda=\alpha$.

\section{Reduction of Theorem 1.3 to the study of singular numbers of some integral operators}

\subsection{Preliminary facts about compact operators}

Let $\mathfrak{H}_{1}$ and $\mathfrak{H}_{2}$ be separable Hilbert spaces. The symbol $\mathfrak{S}_{\infty}\left(\mathfrak{H}_{1}, \mathfrak{H}_{2}\right)$ stands for the class of compact linear operators acting from $\mathfrak{H}_{1}$ to $\mathfrak{H}_{2}\left(\right.$ if $\mathfrak{H}_{1}=\mathfrak{H}_{2}=\mathfrak{H}$, we use the notation $\mathfrak{S}_{\infty}(\mathfrak{H})$, as above). For any $T \in \mathfrak{S}_{\infty}\left(\mathfrak{H}_{1}, \mathfrak{H}_{2}\right)$, by $s_{n}(T), n \in \mathbb{N}$, we denote the singular numbers of the operator $T$ (i. e., the consecutive eigenvalues of the operator $\left(T^{*} T\right)^{1 / 2}$ ). The counting function of singular numbers is defined as follows:

$$
n(s, T):=\#\left\{n \in \mathbb{N}: s_{n}(T)>s\right\}, \quad s>0, \quad T \in \mathfrak{S}_{\infty}\left(\mathfrak{H}_{1}, \mathfrak{H}_{2}\right) .
$$

Obviously, we have

$$
\begin{gathered}
n(s, T)=n_{+}(s, T)+n_{-}(s, T), \quad s>0, \quad T=T^{*}, \\
n(s, T)=n_{+}(s, T), \quad s>0, \quad T \geqslant 0, \\
n\left(s, T^{*} T\right)=n(\sqrt{s}, T)=n\left(\sqrt{s}, T^{*}\right)=n\left(s, T T^{*}\right), \quad s>0 .
\end{gathered}
$$

We shall need the following "variational" property of the counting function $n_{+}(s, T)$ for a selfadjoint compact operator $T$ in the Hilbert space $\mathfrak{H}$ (see, e. g., [9], Ch. 10, Sec. 2, Subsec. 2):

$$
n_{+}(s, T)=\sup \left\{\operatorname{dim} \mathcal{F}, \mathcal{F} \subset \mathfrak{H}:(T u, u)>s\|u\|^{2}, \forall u \in \mathcal{F} \backslash\{0\}\right\} .
$$

Let $\Sigma_{q}\left(\mathfrak{H}_{1}, \mathfrak{H}_{2}\right), q>0$, be the class of compact operators $T$ such that the following functional is finite:

$$
\|T\|_{\Sigma_{q}}:=\sup _{s>0} s n^{1 / q}(s, T),
$$

(see, e. g., [5], [10]). Functional (2.3) is a quasinorm; the space $\Sigma_{q}$ is complete with respect to the quasinorm (2.3) and, in general, nonseparable. We define the following continuous functionals on $\Sigma_{q}$ (see, e. g., [11]):

$$
\begin{aligned}
\Delta_{q}(T) & :=\limsup _{s \rightarrow+0} s^{q} n(s, T), \\
\delta_{q}(T) & :=\liminf _{s \rightarrow+0} s^{q} n(s, T) .
\end{aligned}
$$


The following functionals are continuous on the class of selfadjoint operators in $\Sigma_{q}$ :

$$
\begin{aligned}
\Delta_{q}^{ \pm}(T) & :=\limsup _{s \rightarrow+0} s^{q} n_{ \pm}(s, T), \\
\delta_{q}^{ \pm}(T) & :=\liminf _{s \rightarrow+0} s^{q} n_{ \pm}(s, T) .
\end{aligned}
$$

Sometimes, we shall use the notation $D_{q}(T)$ for any of functionals $\Delta_{q}(T), \Delta_{q}^{ \pm}(T), \delta_{q}(T)$, $\delta_{q}^{ \pm}(T)$; in the case $D_{q}(T)=\Delta_{q}^{ \pm}(T), \delta_{q}^{ \pm}(T)$ it is automatically subtended that $T=T^{*}$. Let $\Sigma_{q}^{0}$ denote the class of operators in $\Sigma_{q}$ such that $\Delta_{q}(T)=0$. Then $\Sigma_{q}^{0}$ is a separable subspace of $\Sigma_{q}$, and the class of finite rank operators is dense in $\Sigma_{q}^{0}$. We note that (see, e. g., [11])

$$
D_{q}(T+K)=D_{q}(T), \quad D=\Delta, \Delta^{ \pm}, \delta, \delta^{ \pm}, \quad T \in \Sigma_{q}, \quad K \in \Sigma_{q}^{0} .
$$

We shall also need the following statement (see, e. g., [9], Ch. 11, Sec. 6, Subsec. 3).

Proposition 2.1. If $T_{1} \in \Sigma_{q_{1}}, T_{2} \in \Sigma_{q_{2}}$, then $T_{1} T_{2} \in \Sigma_{q}$, where $q^{-1}=q_{1}^{-1}+q_{2}^{-1}$, and

$$
\left\|T_{1} T_{2}\right\|_{\Sigma_{q}} \leqslant C\left(q_{1}, q_{2}\right)\left\|T_{1}\right\|_{\Sigma_{q_{1}}}\left\|T_{2}\right\|_{\Sigma_{q_{2}}}, \quad q^{-1}=q_{1}^{-1}+q_{2}^{-1} .
$$

If, additionally, $T_{1} \in \Sigma_{q_{1}}^{0}$ or $T_{2} \in \Sigma_{q_{2}}^{0}$, then $T_{1} T_{2} \in \Sigma_{q}^{0}$.

Finally, if $T \in \Sigma_{q}$ and $A, B$ are bounded operators, then $A T B \in \Sigma_{q}$, and

$$
\|A T B\|_{\Sigma_{q}} \leqslant\|A\|\|B\|\|T\|_{\Sigma_{q}}
$$

\subsection{The study of the bordered resolvent}

By Proposition 1.1, in order to prove asymptotics (1.15) for $\lambda \in(\alpha, \beta)$, it suffices to show that

$$
\lim _{s \rightarrow+0} s^{q} n_{+}(s, Z(\lambda))=\Gamma_{\varrho}(\lambda), \quad \lambda \in(\alpha, \beta), \quad q \varrho=d .
$$

We introduce some notation in order to represent the bordered resolvent $Z(\lambda), \lambda \in(\alpha, \beta)$, as a sum of two terms which correspond to different parts of the spectrum of $A$. We put

$$
\begin{gathered}
m_{A}:=\inf \sigma(A), \quad E_{1}:=E_{A}\left(\left[m_{A}, \alpha\right]\right), \quad E_{2}:=E_{A}([\beta,+\infty)) \\
\mathfrak{H}_{j}:=E_{j} \mathfrak{H}, \quad A_{j}:=\left.A\right|_{\mathfrak{H}_{j}}, \quad I_{j}:=\left.I\right|_{\mathfrak{H}_{j}}, \quad j=1,2 ; \\
Z_{j}(\lambda):=\sqrt{V} E_{j}\left(\lambda I_{j}-A_{j}\right)^{-1} E_{j} \sqrt{V}, \quad j=1,2, \quad \lambda \in(\alpha, \beta) .
\end{gathered}
$$

By (1.11), the operators $Z_{j}(\lambda)$ are compact for $j=1,2$ and $\lambda \in(\alpha, \beta)$. Obviously, $Z(\lambda)=$ $Z_{1}(\lambda)+Z_{2}(\lambda), \lambda \in(\alpha, \beta)$. Theorem 1.3 is a consequence of the following lemma.

Lemma 2.2. Suppose that conditions (1.1), (1.2), (1.8), (1.9), and (1.10) are satisfied. Let $\varrho \in(0, d), d \geqslant 1$, and $q \varrho=d$. Then we have

$$
Z_{1}(\lambda) \in \Sigma_{q}(\mathfrak{H}), \quad \lambda \in(\alpha, \beta)
$$




$$
\begin{gathered}
E_{1} Z_{2}(\lambda) E_{1} \in \Sigma_{q}^{0}(\mathfrak{H}), \quad \lambda \in(\alpha, \beta) \\
\delta_{q}^{+}\left(E_{1} Z_{1}(\lambda) E_{1}\right)=\Delta_{q}^{+}\left(Z_{1}(\lambda)\right)=\Gamma_{\varrho}(\lambda), \quad \lambda \in(\alpha, \beta) .
\end{gathered}
$$

Under the additional condition (1.14), we have

$$
Z_{1}(\lambda) \leqslant Z_{1}(\alpha), \quad \lambda \in(\alpha, \beta)
$$

where $Z_{1}(\alpha)$ is a compact operator such that

$$
Z_{1}(\alpha) \in \Sigma_{q}(\mathfrak{H}), \quad \Delta_{q}^{+}\left(Z_{1}(\alpha)\right)=\Gamma_{\varrho}(\alpha) .
$$

In the end of the present section, the proof of Lemma 2.2 will be reduced to Proposition 2.3 about asymptotics of singular numbers of some integral operators. Now we show how Theorem 1.3 follows from Lemma 2.2.

\subsection{Deduction of Theorem 1.3 from Lemma 2.2}

In the sum $Z(\lambda)=Z_{1}(\lambda)+Z_{2}(\lambda), \lambda \in(\alpha, \beta)$, the second term is negative, whence

$$
n_{+}(s, Z(\lambda)) \leqslant n_{+}\left(s, Z_{1}(\lambda)\right), \quad \lambda \in(\alpha, \beta), \quad s>0 .
$$

From (2.10) and (2.13) we obtain the upper estimate:

$$
\limsup _{s \rightarrow+0} s^{q} n_{+}(s, Z(\lambda)) \leqslant \Gamma_{\varrho}(\lambda), \quad \lambda \in(\alpha, \beta), \quad q \varrho=d .
$$

The variational property (2.2) implies the lower estimate:

$$
n_{+}(s, Z(\lambda)) \geqslant n_{+}\left(s, E_{1} Z(\lambda) E_{1}\right), \quad \lambda \in(\alpha, \beta), \quad s>0 .
$$

Since $E_{1} Z(\lambda) E_{1}=E_{1} Z_{1}(\lambda) E_{1}+E_{1} Z_{2}(\lambda) E_{1}, \lambda \in(\alpha, \beta)$, from (2.8) and (2.9) it follows that $E_{1} Z(\lambda) E_{1} \in \Sigma_{q}, \lambda \in(\alpha, \beta), q \varrho=d$. Taking (2.4), (2.9), and (2.10) into account, we obtain

$$
\delta_{q}^{+}\left(E_{1} Z(\lambda) E_{1}\right)=\Gamma_{\varrho}(\lambda), \quad \lambda \in(\alpha, \beta), \quad q \varrho=d .
$$

From (2.15) and (2.16) it follows that

$$
\liminf _{s \rightarrow+0} s^{q} n_{+}(s, Z(\lambda)) \geqslant \Gamma_{\varrho}(\lambda), \quad \lambda \in(\alpha, \beta), \quad q \varrho=d .
$$

Inequalities (2.14) and (2.17) imply asymptotics (2.6) and asymptotics (1.15) for $\lambda \in(\alpha, \beta)$ with it. It remains to note that, under condition (1.14), relations (1.13), (2.11)-(2.13) imply that $N(\alpha, \tau, V)$ is finite for all $\tau>0$, and

$$
\limsup _{\tau \rightarrow+\infty} \tau^{-d / \varrho} N(\alpha, \tau, V) \leqslant \Gamma_{\varrho}(\alpha) .
$$

From the other side, by monotonicity of $N(\lambda, \tau, V)$, we have

$$
\liminf _{\tau \rightarrow+\infty} \tau^{-d / \varrho} N(\alpha, \tau, V) \geqslant \Gamma_{\varrho}(\lambda), \quad \lambda \in(\alpha, \beta) .
$$

Passing to the limit in (2.19) as $\lambda \rightarrow \alpha+0$ and comparing the result with (2.18), we see that, under the additional condition (1.14), asymptotics (1.15) is valid also in the point $\lambda=\alpha$. 


\subsection{Asymptotics of singular numbers for integral operators of special form}

Here we formulate the result about integral operators which is required for the proof of Lemma 2.2. Suppose that $U(x)$ is a measurable function in $\mathbb{R}^{d}$ satisfying the following condition

$$
U \in L_{\infty}\left(\mathbb{R}^{d}\right), \quad U(x)=v(x /|x|)|x|^{-\nu}+o\left(|x|^{-\nu}\right), \quad|x| \rightarrow \infty, \quad \nu>0 .
$$

Note that under these assumptions $v$ is bounded on $\mathbb{S}^{d-1}$. Assume that functions $f(k), h(k)$, $k \in \widetilde{\Omega}$, satisfy the following condition:

$$
f, h \in L_{2 \varkappa}(\widetilde{\Omega}), \quad \varkappa \nu=d .
$$

Let $\Phi_{s}$ be the operators (1.6). Consider the operators

$$
X_{s t}:=[f(k)] \Phi_{s}[U(x)] \Phi_{t}^{*}[h(k)], \quad s, t \in \mathbb{N} .
$$

In Section 3, we shall prove the following statement.

Proposition 2.3. Suppose that conditions (1.1), (1.2), (2.20), and (2.21) are satisfied. Let $\nu \in(0, d)$ and $\varkappa \nu=d$. Then $X_{s t} \in \Sigma_{\varkappa}$ and

$$
\delta_{\varkappa}\left(X_{s t}\right)=\Delta_{\varkappa}\left(X_{s t}\right)=d^{-1}(2 \pi)^{-d} \delta_{s t} \int_{\widetilde{\Omega}}|f(k) h(k)|^{\varkappa} d k \int_{\mathbb{S}^{d-1}}|v(\theta)|^{\varkappa} d S(\theta), \quad s, t \in \mathbb{N} .
$$

A similar statement was discussed before (without proof) in [8].

Now, we assume that condition $(2.20)$ is satisfied with some $\nu \in(0, d)$. Assume also that

$$
f_{s}, h_{s} \in L_{2 \varkappa}(\widetilde{\Omega}), \quad \varkappa \nu=d, \quad s=1, \ldots, l-1 .
$$

By Proposition 2.3, we have

$$
Y:=\sum_{s, t=1}^{l-1} \Phi_{t}^{*}\left[f_{t}(k)\right] \Phi_{t}[U(x)] \Phi_{s}^{*}\left[h_{s}(k)\right] \Phi_{s} \in \Sigma_{\varkappa} .
$$

By (2.23), the terms $\Phi_{t}^{*}\left[f_{t}(k)\right] \Phi_{t}[U(x)] \Phi_{s}^{*}\left[h_{s}(k)\right] \Phi_{s}$ with $t \neq s$ belong to the class $\Sigma_{\varkappa}^{0}$. Hence, taking (2.4) into account, we have

$$
\delta_{\varkappa}(Y)=\delta_{\varkappa}\left(Y_{0}\right), \quad \Delta_{\varkappa}(Y)=\Delta_{\varkappa}\left(Y_{0}\right),
$$

where

$$
Y_{0}:=\sum_{s=1}^{l-1} \Phi_{s}^{*}\left[f_{s}(k)\right] \Phi_{s}[U(x)] \Phi_{s}^{*}\left[h_{s}(k)\right] \Phi_{s} .
$$


Each operator $\Phi_{s}^{*}\left[f_{s}(k)\right] \Phi_{s}[U(x)] \Phi_{s}^{*}\left[h_{s}(k)\right] \Phi_{s}$ acts nontrivially only in the subspace $\mathfrak{H}_{s}=$ $\Phi_{s}^{*} \Phi_{s} \mathfrak{H}$. The subspaces $\mathfrak{H}_{s}$ are mutually orthogonal. Hence, we have

$$
D_{\varkappa}\left(Y_{0}\right)=\sum_{s=1}^{l-1} D_{\varkappa}\left(\left.\Phi_{s}^{*}\left[f_{s}(k)\right] \Phi_{s}[U(x)] \Phi_{s}^{*}\left[h_{s}(k)\right] \Phi_{s}\right|_{\mathfrak{H}_{s}}\right), \quad D=\Delta, \delta .
$$

The operator $\left.\Phi_{s}^{*}\left[f_{s}(k)\right] \Phi_{s}[U(x)] \Phi_{s}^{*}\left[h_{s}(k)\right] \Phi_{s}\right|_{\mathfrak{H}_{s}}$ is unitarily equivalent to the operator

$$
\left[f_{s}(k)\right] \Phi_{s}[U(x)] \Phi_{s}^{*}\left[h_{s}(k)\right]: L_{2}(\widetilde{\Omega}) \rightarrow L_{2}(\widetilde{\Omega}) .
$$

Relations (2.23), (2.26)-(2.28) imply that

$$
\delta_{\varkappa}(Y)=\Delta_{\varkappa}(Y)=d^{-1}(2 \pi)^{-d} \sum_{s=1}^{l-1} \int_{\widetilde{\Omega}}\left|f_{s}(k) h_{s}(k)\right|^{\varkappa} d k \int_{\mathbb{S}^{d-1}}|v(\theta)|^{\varkappa} d S(\theta) .
$$

Thus, we arrive at the next statement.

Corollary 2.4. Suppose that conditions (1.1), (1.2), (2.20), and (2.24) are satisfied. Let $\nu \in(0, d)$ and $\varkappa \nu=d$. Then relations (2.25) and (2.29) are true.

\subsection{Deduction of Lemma 2.2 from Proposition 2.3 and Corollary 2.4}

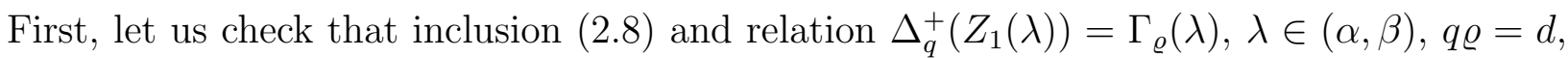
are valid under the assumptions of Lemma 2.2. By (2.1) and (2.7), it suffices to prove that

$$
\begin{gathered}
F(\lambda):=E_{1}\left(\lambda I_{1}-A_{1}\right)^{-1 / 2} E_{1} V E_{1}\left(\lambda I_{1}-A_{1}\right)^{-1 / 2} E_{1} \in \Sigma_{q}, \quad \lambda \in(\alpha, \beta), \quad q \varrho=d, \\
\Delta_{q}(F(\lambda))=\Gamma_{\varrho}(\lambda), \quad \lambda \in(\alpha, \beta), \quad q \varrho=d .
\end{gathered}
$$

By (1.7), the operator $F(\lambda), \lambda \in(\alpha, \beta)$, can be represented as

$$
F(\lambda)=\sum_{s, t=1}^{l-1} \Phi_{t}^{*}\left[\left(\lambda-E_{t}(k)\right)^{-1 / 2}\right] \Phi_{t}[V(x)] \Phi_{s}^{*}\left[\left(\lambda-E_{s}(k)\right)^{-1 / 2}\right] \Phi_{s} .
$$

Relations (2.30) and (2.31) follow from Corollary $2.4\left(\right.$ with $f_{s}(k)=h_{s}(k)=\left(\lambda-E_{s}(k)\right)^{-1 / 2}$, $\left.k \in \widetilde{\Omega}, U(x)=V(x), x \in \mathbb{R}^{d}, \nu=\varrho, \varkappa=q\right)$. Moreover, according to Corollary 2.4, under the additional condition (1.14) the right-hand side of (2.32) with $\lambda=\alpha$ defines an operator $F(\alpha) \in \Sigma_{q}$ such that $\Delta_{q}(F(\alpha))=\Gamma_{\varrho}(\alpha), q \varrho=d$. Consequently, the operator

$$
Z_{1}(\alpha):=\sum_{s=1}^{l-1} \sqrt{V} \Phi_{s}^{*}\left[\left(\alpha-E_{s}(k)\right)^{-1}\right] \Phi_{s} \sqrt{V}
$$

satisfies (2.12) with $q \varrho=d$. Moreover, inequality (2.11) is obvious. 
By $(2.1)$ and $(2.7)$, in order to check that $\delta_{q}^{+}\left(E_{1} Z_{1}(\lambda) E_{1}\right)=\Gamma_{\varrho}(\lambda), \lambda \in(\alpha, \beta), q \varrho=d$, it suffices to prove that

$$
\begin{gathered}
G(\lambda):=E_{1}\left(\lambda I_{1}-A_{1}\right)^{-1 / 2} E_{1} \sqrt{V} E_{1} \in \Sigma_{2 q}, \quad \lambda \in(\alpha, \beta), \quad q \varrho=d, \\
\delta_{2 q}(G(\lambda))=\Gamma_{\varrho}(\lambda), \quad \lambda \in(\alpha, \beta), \quad q \varrho=d .
\end{gathered}
$$

By (1.7), the operator $G(\lambda)$ is represented as

$$
G(\lambda)=\sum_{s, t=1}^{l-1} \Phi_{t}^{*}\left[\left(\lambda-E_{t}(k)\right)^{-1 / 2}\right] \Phi_{t}[\sqrt{V(x)}] \Phi_{s}^{*} \Phi_{s}, \quad \lambda \in(\alpha, \beta) .
$$

Then relations (2.34) and (2.35) follow from Corollary $2.4\left(\right.$ with $f_{s}(k)=\left(\lambda-E_{s}(k)\right)^{-1 / 2}$, $\left.h_{s}(k)=1, k \in \widetilde{\Omega}, U(x)=\sqrt{V(x)}, x \in \mathbb{R}^{d}, \nu=\varrho / 2, \varkappa=2 q\right)$.

Now we check (2.9) with $q \varrho=d$. From Proposition 2.3 (with $f(k)=h(k)=1, k \in \widetilde{\Omega}$, $U(x)=V(x), x \in \mathbb{R}^{d}, \nu=\varrho, \varkappa=q$ ) it follows that $\Phi_{s}[V(x)] \Phi_{s}^{*} \in \Sigma_{q}, q \varrho=d$, whence (see $(2.1),(2.5))$

$$
\sqrt{V} E_{1}=\sum_{s=1}^{l-1} \sqrt{V} \Phi_{s}^{*} \Phi_{s} \in \Sigma_{2 q}, \quad q \varrho=d
$$

Next, the subspaces $\mathfrak{H}_{s}=\Phi_{s}^{*} \Phi_{s} \mathfrak{H}, s \in \mathbb{N}$, are mutually orthogonal, and

$$
\max _{k \in \widetilde{\Omega}}\left|\left(\lambda-E_{s}(k)\right)^{-1}\right| \rightarrow 0, \quad s \rightarrow+\infty .
$$

Hence, the series

$$
\sum_{s=l}^{+\infty} \Phi_{s}^{*}\left[\left(\lambda-E_{s}(k)\right)^{-1}\right] \Phi_{s}=E_{2}\left(\lambda I_{2}-A_{2}\right)^{-1} E_{2}, \quad \lambda \in(\alpha, \beta),
$$

is convergent in the norm of $\mathfrak{B}(\mathfrak{H})$. Combining this with $(2.36)$, we conclude that the series

$$
\sum_{s=l}^{+\infty} E_{1} \sqrt{V} \Phi_{s}^{*}\left[\left(\lambda-E_{s}(k)\right)^{-1}\right] \Phi_{s} \sqrt{V} E_{1}=E_{1} Z_{2}(\lambda) E_{1}, \quad \lambda \in(\alpha, \beta),
$$

is convergent in the class $\Sigma_{q}, q \varrho=d$ (see Proposition 2.1 and (2.5)). From the other side, Proposition 2.3 (with $f(k)=h(k)=1, k \in \widetilde{\Omega}, U(x)=\sqrt{V(x)}, x \in \mathbb{R}^{d}, \nu=\varrho / 2, \varkappa=2 q$ ) implies that

$$
\Phi_{t}[\sqrt{V(x)}] \Phi_{s}^{*} \in \Sigma_{2 q}^{0}, \quad t \neq s .
$$

Consequently, by Proposition 2.1, each term of the series (2.38) belongs to $\Sigma_{q}^{0}, q \varrho=d$. This proves (2.9). 


\section{Estimates and asymptotics of singular numbers of some integral operators}

The present section is devoted to estimates and asymptotics of singular numbers of the integral operators of the form (2.22).

\subsection{Preliminaries. Weak $L_{q}$-classes}

Let $(\mathcal{Z}, d \nu)$ be a separable measurable space with $\sigma$-finite measure. For a $\nu$-measurable function $f: \mathcal{Z} \rightarrow \mathbb{C}$, we put

$$
O_{f}(s):=\{z \in \mathcal{Z}:|f(z)|>s\}, \quad \nu_{f}(s):=\nu\left(O_{f}(s)\right) .
$$

Let $L_{q, \infty}(\mathcal{Z}, d \nu), q>0$, be the class of functions $f$ such that the following functional is finite:

$$
\|f\|_{L_{q, \infty}}:=\sup _{s>0} s \nu_{f}^{1 / q}(s) .
$$

Functional (3.1) is a quasinorm. The space $L_{q, \infty}$ is complete with respect to the quasinorm (3.1). In general, the class $L_{q, \infty}$ is nonseparable. We consider the following subspace of $L_{q, \infty}$ :

$$
L_{q, \infty}^{0,0}(\mathcal{Z}, d \nu):=\left\{f \in L_{q, \infty}: \nu_{f}(s)=o\left(s^{-q}\right), s \rightarrow+0\right\} .
$$

Remark 3.1. 1) Suppose that a function $f \in L_{\infty}\left(\mathbb{R}^{d}\right)$ has the following asymptotics

$$
f(x)=\omega(x /|x|)|x|^{-d / q}+o\left(|x|^{-d / q}\right), \quad|x| \rightarrow \infty, \quad q>0 .
$$

Then $f \in L_{q, \infty}\left(\mathbb{R}^{d}\right)$.

2) Let $f(x):=\omega(x /|x|)|x|^{-d / q}, x \in \mathbb{R}^{d}, q>0$, where $\omega \in L_{q}\left(\mathbb{S}^{d-1}\right)$. Then

$$
f \in L_{q, \infty}\left(\mathbb{R}^{d}\right), \quad\|f\|_{L_{q, \infty}}=d^{-1 / q}\|\omega\|_{L_{q}} .
$$

3) If $f \in L_{q, \infty}\left(\mathbb{R}^{d}\right)$ and $f(x)=o\left(|x|^{-d / q}\right)$ as $|x| \rightarrow \infty, q>0$, then $f \in L_{q, \infty}^{0,0}\left(\mathbb{R}^{d}\right)$.

\subsection{The generalized Cwikel estimate}

Let $(\mathcal{X}, d \varrho)$ and $(\mathcal{Y}, d \tau)$ be two separable spaces with $\sigma$-finite measures. Let $t(x, y), x \in \mathcal{X}$, $y \in \mathcal{Y}$, be the kernel of a bounded integral operator $T$ acting from $L_{2}(\mathcal{Y}, d \tau)$ to $L_{2}(\mathcal{X}, d \varrho)$. Let $w: \mathcal{X} \rightarrow \mathbb{C}$ and $h: \mathcal{Y} \rightarrow \mathbb{C}$ be measurable functions. We consider an integral operator $w T h:$ $L_{2}(\mathcal{Y}, d \tau) \rightarrow L_{2}(\mathcal{X}, d \varrho)$ with the kernel $w(x) t(x, y) h(y)$. We formulate the compactness conditions and estimates of singular numbers for the operator $w T h$ in terms of the function $(w h)(x, y):=w(x) h(y)$ and the measure

$$
d \nu(x, y):=|t(x, y)|^{2} d \varrho(x) d \tau(y), \quad x \in \mathcal{X}, \quad y \in \mathcal{Y} .
$$


The next statement (the generalized Cwikel estimate) was obtained in [12].

Proposition 3.2. Suppose that $w h \in L_{2 q, \infty}(\mathcal{X} \times \mathcal{Y}, d \nu)$ for some $q>1$. Then $w T h \in \Sigma_{2 q}$ and

$$
\|w T h\|_{\Sigma_{2 q}} \leqslant C(q)\|T\|^{1-1 / q}\|w h\|_{L_{2 q, \infty}} .
$$

If $w h \in L_{q, \infty}^{0,0}(\mathcal{X} \times \mathcal{Y}, d \nu), q>1$, then $w T h \in \Sigma_{2 q}^{0}$.

Under the additional assumption that the kernel $t(x, y)$ is bounded, Proposition 3.2 implies the following statement (see [12] and also [13]).

Proposition 3.3. Let $t(\cdot, \cdot) \in L_{\infty}(\mathcal{X} \times \mathcal{Y}, d \varrho d \tau)$. Suppose that $w \in L_{2 q, \infty}(\mathcal{X}, d \varrho)$ and $h \in L_{2 q}(\mathcal{Y}, d \tau)$ for some $q>1$. Then $w T h \in \Sigma_{2 q}$ and

$$
\|w T h\|_{\Sigma_{2 q}} \leqslant C(q)\|t(\cdot, \cdot)\|_{L_{\infty}}^{1 / q}\|T\|^{1-1 / q}\|w\|_{L_{2 q, \infty}}\|h\|_{L_{2 q}} .
$$

If $w \in L_{2 q, \infty}^{0,0}(\mathcal{X}, d \varrho)$ and $h \in L_{2 q}(\mathcal{Y}, d \tau), q>1$, then $w T h \in \Sigma_{2 q}^{0}$.

Obviously, in Proposition 3.3 assumptions on $w$ and $h$ can be interchanged.

\subsection{Asymptotics of singular numbers for some pseudodifferential operators of negative order}

Let $\mathbf{b}(x)=\left(b^{1}(x), b^{2}(x), \ldots, b^{M}(x)\right)$ be a row-matrix and $\mathbf{c}(x)=\left(c^{1}(x), c^{2}(x), \ldots, c^{M}(x)\right)^{t}$ be a column-matrix. Assume that $\mathbf{b}, \mathbf{c} \in L_{2 q}\left(\mathbb{R}^{d}\right), q>1$, and that the matrix-valued functions $\mathbf{b}(x), \mathbf{c}(x)$ are compactly supported. Let $\zeta \in C^{\infty}\left(\mathbb{R}^{d}\right)$ be such that $\zeta(\xi) \geqslant 0$ for $\xi \in \mathbb{R}^{d}$, $\zeta(\xi)=0$ for $|\xi| \leqslant 1$, and $\zeta(\xi)=1$ for $|\xi| \geqslant 2$. Let $\mathbf{v}(\theta)$ be an $(M \times M)$-matrix-valued function on $\mathbb{S}^{d-1}$ such that $\mathbf{v} \in C^{\infty}\left(\mathbb{S}^{d-1}\right)$. We define an $(M \times M)$-matrix-valued function $\mathbf{a}(\xi)$ by the relation

$$
\mathbf{a}(\xi)=\mathbf{v}(\xi /|\xi|)|\xi|^{-d / q} \zeta(\xi), \quad \xi \in \mathbb{R}^{d}, \quad q>1 .
$$

Then the symbol $G(x, \xi)=\mathbf{b}(x) \mathbf{a}(\xi) \mathbf{c}(x)$ is a scalar-valued function in $\mathbb{R}^{d} \times \mathbb{R}^{d}$. Consider the following pseudodifferential operator in $L_{2}\left(\mathbb{R}^{d}\right)$ :

$$
A=\mathbf{b} \Phi \mathbf{a} \Phi^{*} \mathbf{c},
$$

where $\Phi$ is the Fourier operator. By Propositions 2.1, 3.3 and Remark 3.1, we have $A \in \Sigma_{q}$. Next statement gives the asymptotics of singular numbers of the operator (3.3).

Proposition 3.4. Suppose that $\mathbf{a}, \mathbf{b}, \mathbf{c}$ are matrix-valued functions described above. Suppose that the function $\mathbf{a}$ is given by (3.2), and that the functions $\mathbf{b}, \mathbf{c} \in L_{2 q}\left(\mathbb{R}^{d}\right), q>1$, are compactly supported. Then we have

$$
\Delta_{q}(A)=\delta_{q}(A)=d^{-1}(2 \pi)^{-d} \iint_{\mathbb{R}^{d} \times \mathbb{S}^{d-1}}|\mathbf{b}(x) \mathbf{v}(\theta) \mathbf{c}(x)|^{q} d x d S(\theta) .
$$

Proposition 3.4 is a particular case of the more general theorem proved in [14], [15]. 


\subsection{Integral operators of the Fourier-Bloch type}

In order to prove Proposition 2.3, it is convenient to study integral operators of more general type than (2.22). Assume that $\psi: \widetilde{\Omega} \times \mathbb{R}^{d} \rightarrow \mathbb{C}$ is a measurable function satisfying the following conditions

$$
\begin{gathered}
\psi(k, x+n)=e^{i k n} \psi(k, x), \quad n \in \mathbb{Z}^{d} ; \\
\sup _{k \in \widetilde{\Omega}}\|\psi(k, \cdot)\|_{L_{2}(\Omega)}<+\infty .
\end{gathered}
$$

Proposition 3.5. Under conditions (3.4) and (3.5) the function $(2 \pi)^{-d / 2} \psi(k, x)$ is a kernel of a bounded integral operator $T[\psi]: L_{2}(\widetilde{\Omega}) \rightarrow L_{2}\left(\mathbb{R}^{d}\right)$. For all $u \in L_{2}(\widetilde{\Omega})$ the integral

$$
(2 \pi)^{-d / 2} \int_{\widetilde{\Omega}} \psi(k, x) u(k) d k=:(T[\psi] u)(x)
$$

is absolutely convergent for almost every $x \in \mathbb{R}^{d}$. We have

$$
\|T[\psi]\| \leqslant \sup _{k \in \widetilde{\Omega}}\|\psi(k, \cdot)\|_{L_{2}(\Omega)} .
$$

Proof. Let $\Omega_{n}=\Omega+n, n \in \mathbb{Z}^{d}$. By (3.4), (3.5), we have $\psi \in L_{2}\left(\widetilde{\Omega} \times \Omega_{n}\right), n \in \mathbb{Z}^{d}$. Hence, for any $u \in L_{2}(\widetilde{\Omega})$ we obtain

$$
(2 \pi)^{-d / 2} \int_{\widetilde{\Omega}}|\psi(k, x)||u(k)| d k \leqslant(2 \pi)^{-d / 2}\left(\int_{\widetilde{\Omega}}|\psi(k, x)|^{2} d k\right)^{1 / 2}\|u\|_{L_{2}(\widetilde{\Omega})}, \quad \text { a. e. } x \in \mathbb{R}^{d},
$$

which implies that the integral in the left-hand side of (3.6) is absolutely convergent for a. e. $x \in \mathbb{R}^{d}$. From (3.4) it follows that

$$
(T[\psi] u)(x+n)=\int_{\widetilde{\Omega}}(2 \pi)^{-d / 2} e^{i k n} \psi(k, x) u(k) d k, \quad x \in \mathbb{R}^{d} .
$$

Since $\left\{(2 \pi)^{-d / 2} e^{i k n}\right\}_{n \in \mathbb{Z}^{d}}$ is an orthonormal basis in $L_{2}(\widetilde{\Omega})$, relation (3.8) implies that

$$
\sum_{n \in \mathbb{Z}^{d}}|(T[\psi] u)(x+n)|^{2}=\int_{\widetilde{\Omega}}|\psi(k, x)|^{2}|u(k)|^{2} d k, \quad x \in \mathbb{R}^{d} .
$$

Integrating the last identity over the cell $\Omega$, we obtain

$$
\int_{\mathbb{R}^{d}}|(T[\psi] u)(x)|^{2} d x=\int_{\widetilde{\Omega}}\|\psi(k, \cdot)\|_{L_{2}(\Omega)}^{2}|u(k)|^{2} d k .
$$

Relation (3.9) implies estimate (3.7).

In what follows, the operator $T[\psi]$ is called the operator of the Fourier-Bloch type.

Remark 3.6. The function $e^{i k x}$ satisfies conditions (3.4) and (3.5). We have

$$
T\left[e^{i k x}\right]=\Phi^{*} \Pi,
$$

where $\Phi: L_{2}\left(\mathbb{R}^{d}\right) \rightarrow L_{2}\left(\mathbb{R}^{d}\right)$ is the Fourier operator and the operator $\Pi: L_{2}(\widetilde{\Omega}) \rightarrow L_{2}\left(\mathbb{R}^{d}\right)$ acts as extension by zero, i. e., $(\Pi u)(k)=u(k)$ for $k \in \widetilde{\Omega}$ and $(\Pi u)(k)=0$ for $k \in \mathbb{R}^{d} \backslash \widetilde{\Omega}$. 


\subsection{The Cwikel estimate for the bordered operator of the Fourier- Bloch type}

Now we give the compactness condition and estimate for singular numbers of the operator $w T[\psi] h$ in terms of the function $(w h)(x, k):=w(x) h(k)$ and measure $d \nu(x, k):=$ $(2 \pi)^{-d}|\psi(k, x)|^{2} d x d k$ on $\mathbb{R}^{d} \times \widetilde{\Omega}$. The following statement is a consequence of Propositions 3.2 and 3.5 .

Corollary 3.7. If $w h \in L_{2 q, \infty}\left(\mathbb{R}^{d} \times \widetilde{\Omega}, d \nu\right)$ for some $q>1$, then $w T[\psi] h \in \Sigma_{2 q}$ and

$$
\|w T[\psi] h\|_{\Sigma_{2 q}} \leqslant C(q)\left(\sup _{k \in \widetilde{\Omega}}\|\psi(k, \cdot)\|_{L_{2}(\Omega)}\right)^{1-1 / q}\|w h\|_{L_{2 q, \infty}} .
$$

If $w h \in L_{2 q, \infty}^{0,0}\left(\mathbb{R}^{d} \times \widetilde{\Omega}, d \nu\right)$, then $w T[\psi] h \in \Sigma_{2 q}^{0}$.

Now, assume that the bordering functions $w(x), h(k)$ satisfy the conditions

$$
|w(x)| \leqslant A\|x\|^{-d / 2 q}, \quad x \in \mathbb{R}^{d} ; \quad h \in L_{\infty}(\widetilde{\Omega}) .
$$

Here we denote $\|x\|=\max \{1,|x|\}, x \in \mathbb{R}^{d}$.

Proposition 3.8. Assume that conditions (3.4), (3.5), and (3.11) with some $q>0$ are satisfied. Then $w h \in L_{2 q, \infty}\left(\mathbb{R}^{d} \times \widetilde{\Omega}, d \nu\right)$ and

$$
\|w h\|_{L_{2 q, \infty}} \leqslant C(q, d) A\|h\|_{L_{\infty}}\|\psi\|_{L_{2}(\widetilde{\Omega} \times \Omega)}^{1 / q} .
$$

Proof. Obviously, we have

$$
O_{w h}(s) \subset\left\{x \in \mathbb{R}^{d}:\|x\|<\left(s / A\|h\|_{L_{\infty}}\right)^{-2 q / d}\right\} \times \widetilde{\Omega}, \quad s>0 .
$$

Combining this with the inequalities $\|x\| \geqslant 1,\|x\| \geqslant|x|, x \in \mathbb{R}^{d}$, we see that

$$
\begin{aligned}
O_{w h}(s) & =\varnothing, \quad s \geqslant A\|h\|_{L_{\infty}}, \\
O_{w h}(s) \subset B_{R}(0) \times \widetilde{\Omega}, \quad R & =\left(s / A\|h\|_{L_{\infty}}\right)^{-2 q / d}, \quad s \in\left(0, A\|h\|_{L_{\infty}}\right),
\end{aligned}
$$

where $B_{R}(0)=\left\{x \in \mathbb{R}^{d}:|x|<R\right\}$. We denote

$$
Z_{R}:=\left\{n \in \mathbb{Z}^{d}: \Omega_{n} \cap B_{R}(0) \neq \varnothing\right\}, \quad \Omega(R):=\bigcup_{n \in Z_{R}} \Omega_{n} .
$$

Since $B_{R}(0) \subset \Omega(R)$, then (3.14) implies that

$$
O_{w h}(s) \subset \Omega(R) \times \widetilde{\Omega}, \quad R=\left(s / A\|h\|_{L_{\infty}}\right)^{-2 q / d}, \quad s \in\left(0, A\|h\|_{L_{\infty}}\right) .
$$


For $s \in\left(0, A\|h\|_{L_{\infty}}\right)$ it follows from (3.15) that

$$
\begin{array}{r}
\nu_{w h}(s) \leqslant(2 \pi)^{-d} \iint_{\widetilde{\Omega} \times \Omega(R)}|\psi(k, x)|^{2} d k d x=(2 \pi)^{-d} \sum_{n \in Z_{R}} \iint_{\widetilde{\Omega} \times \Omega_{n}}|\psi(k, x)|^{2} d k d x= \\
=(2 \pi)^{-d} \iint_{\widetilde{\Omega} \times \Omega}|\psi(k, x)|^{2} d k d x \cdot \# Z_{R}, \quad R=\left(s / A\|h\|_{L_{\infty}}\right)^{-2 q / d} .
\end{array}
$$

Using obvious relations $\# Z_{R}=\operatorname{Vol} \Omega(R)$ and $\Omega(R) \subset B_{R+\sqrt{d}}(0)$, we obtain

$$
\# Z_{R} \leqslant C(d)(R+\sqrt{d})^{d} .
$$

Combining (3.1), (3.13), (3.16), and (3.17), we arrive at (3.12).

Corollary 3.7 and Proposition 3.8 imply the following statement.

Proposition 3.9. Suppose that conditions (3.4), (3.5), and (3.11) with some $q>1$ are satisfied. Then $w T[\psi] h \in \Sigma_{2 q}$ and

$$
\|w T[\psi] h\|_{\Sigma_{2 q}} \leqslant C(q, d)\left(\sup _{k \in \widetilde{\Omega}}\|\psi(k, \cdot)\|_{L_{2}(\Omega)}\right)^{1-1 / q} A\|h\|_{L_{\infty}}\|\psi\|_{L_{2}(\widetilde{\Omega} \times \Omega)}^{1 / q} .
$$

\subsection{Asymptotics of singular numbers of the product of two bor- dered operators of the Fourier-Bloch type}

Assume that functions $\psi_{j} \in L_{\infty}\left(\widetilde{\Omega} \times \mathbb{R}^{d}\right), j=1,2$, satisfy conditions (3.4), (3.5). Let $U \in L_{\infty}\left(\mathbb{R}^{d}\right)$ be such that

$$
U(x)=v(x /|x|)|x|^{-d / q}+o\left(|x|^{-d / q}\right), \quad|x| \rightarrow+\infty,
$$

for some $q>1$. Note that under assumption (3.18) we have $v \in L_{\infty}\left(\mathbb{S}^{d-1}\right)$. Finally, assume that functions $f, h: \widetilde{\Omega} \rightarrow \mathbb{C}$ satisfy the condition

$$
f, h \in L_{2 q}(\widetilde{\Omega}) .
$$

Consider the following integral operator

$$
X:=[f(k)]\left(T\left[\psi_{1}\right]\right)^{*}[U(x)] T\left[\psi_{2}\right][h(k)] .
$$

We put

$$
G_{q}\left(f, v, h, \psi_{1}, \psi_{2}\right):=d^{-1}(2 \pi)^{-d} \int_{\mathbb{S}^{d-1}}|v(\theta)|^{q} d S(\theta) \int_{\widetilde{\Omega}}\left|h(k) f(k)\left(\psi_{2}(k, \cdot), \psi_{1}(k, \cdot)\right)_{L_{2}(\Omega)}\right|^{q} d k .
$$


Proposition 3.10. Assume that functions $\psi_{j} \in L_{\infty}\left(\widetilde{\Omega} \times \mathbb{R}^{d}\right), j=1,2$, satisfy conditions (3.4) and (3.5). Assume that $U \in L_{\infty}\left(\mathbb{R}^{d}\right)$ satisfies (3.18) for some $q>1$. Let $f, h: \widetilde{\Omega} \rightarrow \mathbb{C}$ be subject to (3.19). Then for the operator (3.20) we have $X \in \Sigma_{q}$ and

$$
\delta_{q}(X)=\Delta_{q}(X)=G_{q}\left(f, v, h, \psi_{1}, \psi_{2}\right) .
$$

The rest of Section 3 is devoted to the proof of Proposition 3.10. Obviously, Proposition 2.3 is a particular case of Proposition 3.10 (with $\psi_{1}=\psi_{s}, \psi_{2}=\psi_{t}, \Phi_{s}=\left(T\left[\psi_{s}\right]\right)^{*}, \Phi_{t}^{*}=T\left[\psi_{t}\right]$, $\varkappa=q, \nu=d / q)$.

\subsection{Proof of Proposition 3.10}

The proof of Proposition 3.10 consists of several steps.

Step 1. By Remark 3.1, we have $U \in L_{q, \infty}\left(\mathbb{R}^{d}\right)$, and the function

$$
R(x):=U(x)-\zeta^{2}(x) v(x /|x|)|x|^{-d / q}=o\left(|x|^{-d / q}\right), \quad|x| \rightarrow \infty,
$$

belongs to the class $L_{q, \infty}^{0,0}\left(\mathbb{R}^{d}\right)$. Here $\zeta(x)$ is the same function as above, i. e., $\zeta \in C^{\infty}\left(\mathbb{R}^{d}\right)$, $\zeta(x) \geqslant 0$ for $x \in \mathbb{R}^{d}, \zeta(x)=0$ for $|x| \leqslant 1$, and $\zeta(x)=1$ for $|x| \geqslant 2$. Obviously, functions $R(x)$ and $\widetilde{U}(x):=\zeta^{2}(x) v(x /|x|)|x|^{-d / q}$ can be represented as

$$
\begin{gathered}
R(x)=R_{1}(x) R_{2}(x), \quad R_{2}(x)=\sqrt{|R(x)|}, \quad R_{1}(x)=e^{i \arg R(x)} R_{2}(x), \quad x \in \mathbb{R}^{d} ; \\
\widetilde{U}(x)=W_{1}(x) W_{2}(x), \quad W_{2}(x)=\zeta(x) \sqrt{|v(x /|x|)|}|x|^{-d / 2 q}, \\
W_{1}(x)=e^{i \arg v(x /|x|)} W_{2}(x), \quad x \in \mathbb{R}^{d} .
\end{gathered}
$$

Then the operator (3.20) can be represented as a sum $X=X^{(1)}+X^{(2)}$, where

$$
\begin{gathered}
X^{(1)}=X_{q}^{(1)}\left(f, v, h, \psi_{1}, \psi_{2}\right)=[f(k)]\left(T\left[\psi_{1}\right]\right)^{*}\left[W_{1}(x)\right]\left[W_{2}(x)\right] T\left[\psi_{2}\right][h(k)], \\
X^{(2)}=[f(k)]\left(T\left[\psi_{1}\right]\right)^{*}\left[R_{1}(x)\right]\left[R_{2}(x)\right] T\left[\psi_{2}\right][h(k)] .
\end{gathered}
$$

Obviously, $W_{j} \in L_{2 q, \infty}\left(\mathbb{R}^{d}\right), R_{j} \in L_{2 q, \infty}^{0,0}\left(\mathbb{R}^{d}\right), j=1,2$. Then, by Propositions 2.1, 3.3, and 3.5, we have $X^{(1)} \in \Sigma_{q}, X^{(2)} \in \Sigma_{q}^{0}$. We have proved the following statement.

Lemma 3.11. Suppose that the assumptions of Proposition 3.10 are satisfied. Let $X$ and $X^{(1)}$ be the operators defined by (3.20) and (3.22) respectively. Then

$$
X, X^{(1)} \in \Sigma_{q}, \quad D_{q}(X)=D_{q}\left(X^{(1)}\right), \quad D=\Delta, \delta .
$$


Step 2. By Remark 3.1, we have

$$
\left\|W_{j}\right\|_{L_{2 q, \infty}} \leqslant C(d, q)\|v\|_{L_{q}}^{1 / 2}, \quad j=1,2 .
$$

From Propositions 2.1, 3.3 and 3.5 and from (3.23) it follows that the operator $X^{(1)}=$ $X_{q}^{(1)}\left(f, v, h, \psi_{1}, \psi_{2}\right)$ (with $q>1$ ) defined by (3.22) belongs to $\Sigma_{q}$ for all $\{f, v, h\} \in L_{2 q}(\widetilde{\Omega}) \times$ $L_{q}\left(\mathbb{S}^{d-1}\right) \times L_{2 q}(\widetilde{\Omega})$. Herewith, the functionals $\Delta_{q}\left(X^{(1)}\right)$ and $\delta_{q}\left(X^{(1)}\right)$ depend on coefficients $\{f, v, h\}$ continuously in the space $\mathcal{L}:=L_{2 q}(\widetilde{\Omega}) \times L_{q}\left(\mathbb{S}^{d-1}\right) \times L_{2 q}(\widetilde{\Omega})$. Similarly, the right-hand side of (3.21) is continuous with respect to $\{f, v, h\}$ in $\mathcal{L}$. Consequently, in order to prove that

$$
D_{q}\left(X_{q}^{(1)}\left(f, v, h, \psi_{1}, \psi_{2}\right)\right)=G_{q}\left(f, v, h, \psi_{1}, \psi_{2}\right), \quad D=\Delta, \delta,
$$

for all $\{f, v, h\} \in \mathcal{L}$, it suffices to check this relation for $\{f, v, h\} \in \mathcal{L}_{0}:=C_{0}^{\infty}(\widetilde{\Omega}) \times C^{\infty}\left(\mathbb{S}^{d-1}\right) \times$ $C_{0}^{\infty}(\widetilde{\Omega})$, since $\mathcal{L}_{0}$ is dense in $\mathcal{L}$. Thus, we have proved the following statement.

Lemma 3.12. Suppose that functions $\psi_{j} \in L_{\infty}\left(\widetilde{\Omega} \times \mathbb{R}^{d}\right), j=1,2$, satisfy conditions (3.4) and (3.5). Let $X^{(1)}=X_{q}^{(1)}\left(f, v, h, \psi_{1}, \psi_{2}\right)$ be the operator (3.22) with $q>1$. Suppose that relation (3.24) is valid for all $\{f, v, h\} \in \mathcal{L}_{0}$. Then relation (3.24) is true for all $\{f, v, h\} \in \mathcal{L}$.

Step 3. Suppose that assumptions of Proposition 3.10 are satisfied. Suppose also that $f, h \in C_{0}^{\infty}(\widetilde{\Omega})$. We put

$$
\varphi_{j}(k, x):=e^{-i k x} \psi_{j}(k, x), \quad k \in \widetilde{\Omega}, \quad x \in \mathbb{R}^{d}, \quad j=1,2 .
$$

By (3.4), the functions $\varphi_{j}(k, \cdot), j=1,2$, are $\mathbb{Z}^{d}$-periodic. We decompose the functions $\varphi_{j}(k, \cdot), j=1,2$, in the Fourier series with respect to the system $\left\{e^{2 \pi i n x}\right\}_{n \in \mathbb{Z}^{d}}$ in $L_{2}(\Omega)$ for a fixed $k \in \widetilde{\Omega}$. Let $\left\{c_{j}^{n}(k)\right\}_{n \in \mathbb{Z}^{d}}$ be the corresponding Fourier coefficients. Then we have

$$
\varphi_{j}(k, x)=\sum_{n \in \mathbb{Z}^{d}} c_{j}^{n}(k) e^{2 \pi i n x}, \quad c_{j}^{n}(k)=\int_{\Omega} \varphi_{j}(k, x) e^{-2 \pi i n x} d x, \quad k \in \widetilde{\Omega}, \quad j=1,2 .
$$

Relations (3.25) can be rewritten as

$$
\psi_{j}(k, x)=e^{i k x} \sum_{n \in \mathbb{Z}^{d}} c_{j}^{n}(k) e^{2 \pi i n x}, \quad c_{j}^{n}(k)=\int_{\Omega} \psi_{j}(k, x) e^{-i k x} e^{-2 \pi i n x} d x, \quad k \in \widetilde{\Omega}, \quad j=1,2 .
$$

We denote

$$
\psi_{j}^{N}(k, x):=e^{i k x} \sum_{|n| \leqslant N} c_{j}^{n}(k) e^{2 \pi i n x}, \quad \Phi_{j}^{N}(k, x):=e^{i k x} \sum_{|n|>N} c_{j}^{n}(k) e^{2 \pi i n x}, \quad k \in \widetilde{\Omega}, \quad j=1,2 .
$$


It is easily seen that the functions $\psi_{j}^{N}(k, x)$ and $\Phi_{j}^{N}(k, x), j=1,2$, satisfy conditions (3.4), (3.5), and

$$
\begin{gathered}
\sup _{k \in \widetilde{\Omega}}\left\|\psi_{j}^{N}(k, \cdot)\right\|_{L_{2}(\Omega)}=\sup _{k \in \widetilde{\Omega}}\left(\sum_{|n| \leqslant N}\left|c_{j}^{n}(k)\right|^{2}\right)^{1 / 2} \leqslant \sup _{k \in \widetilde{\Omega}}\left\|\psi_{j}(k, \cdot)\right\|_{L_{2}(\Omega)} ; \\
\sup _{k \in \widetilde{\Omega}}\left\|\Phi_{j}^{N}(k, \cdot)\right\|_{L_{2}(\Omega)}=\sup _{k \in \widetilde{\Omega}}\left(\sum_{|n|>N}\left|c_{j}^{n}(k)\right|^{2}\right)^{1 / 2} \leqslant \sup _{k \in \widetilde{\Omega}}\left\|\psi_{j}(k, \cdot)\right\|_{L_{2}(\Omega)} ; \\
\left\|\Phi_{j}^{N}\right\|_{L_{2}(\widetilde{\Omega} \times \Omega)}^{2}=\int_{\widetilde{\Omega}} d k \sum_{|n|>N}\left|c_{j}^{n}(k)\right|^{2} \rightarrow 0, \quad N \rightarrow \infty .
\end{gathered}
$$

The last relation follows by the Lebesgue theorem from (3.29) and from the convergence $\sum_{|n|>N}\left|c_{j}^{n}(k)\right|^{2} \rightarrow 0$ as $N \rightarrow \infty$ for all $k \in \widetilde{\Omega}$. Under the assumptions of Proposition 3.10 the function $v(\theta), \theta \in \mathbb{S}^{d-1}$, is bounded, whence the pairs of functions $W_{1}, f$ and $W_{2}, h$ satisfy condition (3.11) for $q>1$. Hence, from (3.29), (3.30), and Proposition 3.9 it follows that $f\left(T\left[\Phi_{1}^{N}\right]\right)^{*} W_{1} \in \Sigma_{2 q}, W_{2} T\left[\Phi_{2}^{N}\right] h \in \Sigma_{2 q}$, and

$$
\begin{gathered}
\left\|f\left(T\left[\psi_{1}\right]\right)^{*} W_{1}-f\left(T\left[\psi_{1}^{N}\right]\right)^{*} W_{1}\right\|_{\Sigma_{2 q}} \rightarrow 0, \quad N \rightarrow \infty ; \\
\left\|W_{2} T\left[\psi_{2}\right] h-W_{2} T\left[\psi_{2}^{N}\right] h\right\|_{\Sigma_{2 q}} \rightarrow 0, \quad N \rightarrow \infty .
\end{gathered}
$$

Combining the last relations with Proposition 2.1, we see that

$$
\left\|X_{q}^{(1)}\left(f, v, h, \psi_{1}^{N}, \psi_{2}^{N}\right)-X_{q}^{(1)}\left(f, v, h, \psi_{1}, \psi_{2}\right)\right\|_{\Sigma_{q}} \rightarrow 0, \quad N \rightarrow \infty .
$$

Obviously, we have

$$
G_{q}\left(f, v, h, \psi_{1}^{N}, \psi_{2}^{N}\right) \rightarrow G_{q}\left(f, v, h, \psi_{1}, \psi_{2}\right), \quad N \rightarrow \infty .
$$

We arrive at the following statement.

Lemma 3.13. Suppose that the assumptions of Proposition 3.10 are satisfied. Let $f, h \in$ $C_{0}^{\infty}(\widetilde{\Omega})$. Suppose also that the following relations are true:

$$
D_{q}\left(X_{q}^{(1)}\left(f, v, h, \psi_{1}^{N}, \psi_{2}^{N}\right)\right)=G_{q}\left(f, v, h, \psi_{1}^{N}, \psi_{2}^{N}\right), \quad D=\Delta, \delta, \quad N \in \mathbb{N} .
$$

Then relation (3.24) is valid.

Step 4. Now, suppose that the assumptions of Proposition 3.10 and also condition $v \in$ $C^{\infty}\left(\mathbb{S}^{d-1}\right)$ are satisfied. Comparing (3.10), (3.22), and (3.27), we can write the operator $X_{q}^{(1)}\left(f, v, h, \psi_{1}^{N}, \psi_{2}^{N}\right)$ as

$$
X_{q}^{(1)}\left(f, v, h, \psi_{1}^{N}, \psi_{2}^{N}\right)=\sum_{|m|,|n| \leqslant N}\left[f(k) \overline{c_{1}^{m}(k)}\right] P \Phi\left[e^{2 \pi i(n-m) x} W_{1}(x) W_{2}(x)\right] \Phi^{*} \Pi\left[h(k) c_{2}^{n}(k)\right] .
$$


Here $P$ is the orthogonal projection of $L_{2}\left(\mathbb{R}^{d}\right)$ onto $L_{2}(\widetilde{\Omega})$ (the operator of restriction to $\widetilde{\Omega}$ ) and, as above, $\Pi: L_{2}(\widetilde{\Omega}) \rightarrow L_{2}\left(\mathbb{R}^{d}\right)$ is the operator of extension by zero from $\widetilde{\Omega}$ to the whole $\mathbb{R}^{d}$. Note that, by (3.19) and (3.28), the functions $f(k) \overline{c_{1}^{m}(k)}, h(k) c_{2}^{n}(k), k \in \widetilde{\Omega},|m|,|n| \leqslant N$, belong to $L_{2 q}(\widetilde{\Omega})$.

Next, we extend the functions $c_{j}^{n}(k), n \in \mathbb{Z}^{d}, j=1,2, f(k), h(k)$, by zero from $\widetilde{\Omega}$ to the whole $\mathbb{R}^{d}$. It is easy to check the following statement.

Remark 3.14. The nonzero singular numbers of the operator $X_{q}^{(1)}\left(f, v, h, \psi_{1}^{N}, \psi_{2}^{N}\right)$ are the same as for the operator

$$
Y_{N}:=\sum_{|m|,|n| \leqslant N}\left[f(k) \overline{c_{1}^{m}(k)}\right] \Phi\left[e^{2 \pi i(n-m) x} W_{1}(x) W_{2}(x)\right] \Phi^{*}\left[h(k) c_{2}^{n}(k)\right]
$$

acting in $L_{2}\left(\mathbb{R}^{d}\right)$.

We define the following shift operator in $L_{2}\left(\mathbb{R}^{d}\right):\left(T_{p} u\right)(x)=u(x-p), \quad p \in \mathbb{R}^{d}$. By the obvious identity $T_{p} \Phi=\Phi\left[e^{i p x}\right], p \in \mathbb{R}^{d}$, the operator $Y_{N}$ can be written as

$$
Y_{N}=\sum_{|m|,|n| \leqslant N} T_{2 \pi(n-m)}\left[f(k+2 \pi(n-m)) \overline{c_{1}^{m}(k+2 \pi(n-m))}\right] \Phi\left[W_{1}(x) W_{2}(x)\right] \Phi^{*}\left[h(k) c_{2}^{n}(k)\right] .
$$

From Proposition 3.4 it follows that the terms in (3.32) with $m \neq n$ belong to $\Sigma_{q}^{0}$. Hence (see $(2.4))$,

$$
\begin{gathered}
\delta_{q}\left(Y_{N}\right)=\delta_{q}\left(Y_{N}^{0}\right), \quad \Delta_{q}\left(Y_{N}\right)=\Delta_{q}\left(Y_{N}^{0}\right), \\
Y_{N}^{0}:=\sum_{|n| \leqslant N}\left[f(k) \overline{c_{1}^{n}(k)}\right] \Phi\left[W_{1}(x) W_{2}(x)\right] \Phi^{*}\left[h(k) c_{2}^{n}(k)\right] .
\end{gathered}
$$

The coefficients $\left\{c_{j}^{n}(k)\right\}$ are enumerated by the multidimensional index $n \in \mathbb{Z}^{d}$. Now it is convenient to renumber the set $\left\{c_{j}^{n}(k)\right\}_{|n| \leqslant N}$ somehow by one number $l=1, \ldots, M$. Then $\left\{c_{j}^{n}(k)\right\}_{|n| \leqslant N}=\left\{c_{j}^{(1)}(k), c_{j}^{(2)}(k), \ldots, c_{j}^{(M)}(k)\right\}, j=1,2$. We put

$$
\begin{gathered}
\mathbf{b}(k):=\left(f(k) \overline{c_{1}^{(1)}(k)}, \ldots, f(k) \overline{c_{1}^{(M)}(k)}\right), \quad \mathbf{c}(k):=\left(h(k) c_{2}^{(1)}(k), \ldots, h(k) c_{2}^{(M)}(k)\right)^{t}, \\
\mathbf{a}(x):=W_{1}(x) W_{2}(x) E,
\end{gathered}
$$

where $E$ is the identity $(M \times M)$-matrix. Then the operator $Y_{N}^{0}$ takes the form (3.3). From Proposition 3.4 it follows that

$$
\delta_{q}\left(Y_{N}^{0}\right)=\Delta_{q}\left(Y_{N}^{0}\right)=G_{q}\left(f, v, h, \psi_{1}^{N}, \psi_{2}^{N}\right) .
$$

Taking Remark 3.14 into account, we arrive at the following statement.

Lemma 3.15. Suppose that the assumptions of Proposition 3.10 and also condition $v \in$ $C^{\infty}\left(\mathbb{S}^{d-1}\right)$ are satisfied. Then relations (3.31) are valid.

Now, Proposition 3.10 follows from Lemmas 3.11, 3.12, 3.13, and 3.15. 


\section{Acknowledgement}

The paper was finished after the death of M. Sh. Birman. I express my deep gratitude to T. A. Suslina who helped me to prepare the final version of the manuscript and translated it into English. I also thank N. D. Filonov who has read the manuscript and made useful remarks.

V. A. Sloushch

\section{References}

[1] M. Sh. Birman. The discrete spectrum of the periodic Schrödinger operator perturbed by a decreasing potential. (Russian) Algebra i Analiz 8 (1996), no. 1, 3-20; English transl., St. Petersburg Math. J. 8 (1997), no. 1, 1-14.

[2] M. Reed, B. Simon. Methods of modern mathematical physics. IV: Analysis of operators. Academic Press, New York, 1978.

[3] M. M. Skriganov. Geometric and arithmetic methods in the spectral theory of multidimensional periodic operators. (Russian) Trudy Mat. Inst. Steklov, vol. 171, 1985, 171 pp. English transl., Proc. Steklov Inst. Math., 1987, no. 2, 121 pp.

[4] M. Sh. Birman. The discrete spectrum in gaps of the perturbed periodic Schrödinger operator. I. Regular perturbations. Boundary value problems, Schrödinger operators, deformation quantization, pp. 334-352, Math. Top., 8, Akademie Verlag, Berlin, 1995.

[5] M. Sh. Birman, G. E. Karadzhov, M. Z. Solomyak. Boundedness conditions and spectrum estimates for the operators $b(X) a(D)$ and their analogs. Estimates and asymptotics for discrete spectra of integral and differential equations. Adv. Soviet Math., vol. 7, Amer. Math. Soc., Providence, RI, 1991, pp. 85-106.

[6] M. Sh. Birman. Discrete spectrum in the gaps of a continuous one for perturbation with large coupling constant. Estimates and asymptotics for discrete spectra of integral and differential equations. Adv. Soviet Math., vol. 7, Amer. Math. Soc., Providence, RI, 1991, pp. 57-73.

[7] S. Alama, P. A. Deift, R. Hempel. Eigenvalue branches of the Schrödinger operator $H-\lambda W$ in a gap of $\sigma(H)$. Commun. Math. Phys. 121 (1989), 291-321.

[8] M. Sh. Birman. Discrete spectrum of the periodic Schrödinger operator for non-negative perturbations. Mathematical results in quantum mechanics (Blossin, 1993), 3-7. Oper. Theory Adv. Appl., Vol. 70, Birkhäuser, Basel, 1994.

[9] M. Sh. Birman, M. Z. Solomyak. Spectral theory of selfadjoint operators in Hilbert space. D. Reidel Publishing Company, 1987, Dordrecht, Holland. 
[10] M. Sh. Birman, M. Z. Solomyak. Estimates for the singular numbers of integral operators. (Russian) Uspekhi Mat. Nauk 32 (1977), no. 1(193), 17-84. English transl., Russian Math. Surveys 32, no. 1 (1977), 15-89.

[11] M. Sh. Birman, M. Z. Solomyak. Compact operators with power-like asymptotics of singular numbers. (Russian) Investigations on linear operators and the theory of functions, 12. Zap. Nauchn. Sem. Leningrad. Otdel. Mat. Inst. Steklov (LOMI) 126 (1983), 21-30. English transl., J. Soviet Math. 27 (1984), 2442-2447.

[12] V. A. Sloushch. Generalizations of the Cwikel estimate for integral operators. (Russian) Trudy Sankt-Peterburgskogo mat. obshchestva, vol. 14 (2008), 169-196. English transl., Proc. St. Petersburg Math. Soc., vol. XIV, Amer. Math. Soc. Transl. (2), vol. 228, 2009.

[13] M. Sh. Birman, M. Z. Solomyak. Negative discrete spectrum of the Schrödinger operator with large coupling constant: a qualitative discussion. Order, disorder, and chaos in quantum systems (Dubna, 1989). Oper. Theory Adv. Appl., vol. 46, Birkhäuser, Basel, 1990, pp. 3-16.

[14] M. Sh. Birman, M. Z. Solomyak. Asymptotic behavior of the spectrum of pseudodifferential operators with anisotropically homogeneous symbols. (Russian) Vestnik Leningrad. Univ. Mat. Mekh. Astronom. 13, no. 3 (1977), 13-21. English transl., Vestnik Leningrad Univ. Math. 10 (1982), 237-247.

[15] M. Sh. Birman, M. Z. Solomyak. Asymptotic behavior of the spectrum of pseudodifferential operators with anisotropically homogeneous symbols. II. (Russian) Vestnik Leningrad. Univ. Mat. Mekh. Astronom. 13, no. 3 (1979), 5-10. English transl., Vestnik Leningrad Univ. Math. 12 (1980), 155-161. 